\title{
A Fixed Interface Boundary Value Problem For Differential Equations: A Problem Arising From Population Genetics
}

\author{
Chi-Kan Chen
}

\author{
Communicated by $Y$. Charles Li, received February 23, 2006 \\ and, in revised form, June 24, 2006.
}

\begin{abstract}
Diffusion type fixed interface conditions are formulated to describe the transport of diffusing materials across porous thin barriers embedded in media supporting the diffusion process. We consider a Neumann boundary value problem with fixed interface conditions for general diffusion-reaction differential equations that models the gene dispersal in a population under natural selection in a finite habitat with embedded narrow barriers. We establish for the problem a new comparison principle, the global existence of solutions, and sufficient conditions of stability and instability of equilibria. We show that the stability of equilibrium changes as the barrier permeability changes through a critical value. Also, the nonconstant stable equilibria for the problem can arise due to the interaction of the selection force and the barrier. Results in this work are applicable to general situations of materials or heat diffusing through permeable barriers.
\end{abstract}

\section{Contents}

1. Introduction 199

2. Bounded Global Solutions 201

3. Stability and Instability of Equilibria 203

4. Application 204

5. Discussion 206

6. Appendix 207

References 208

\section{Introduction}

Genetic materials (genes) disperse in spatially distributed populations subject to the joint action of evolutionary forces such as migration and selection. Recent study has shown that regions in the population's habitat of geographical obstacles, reduced population density, or spatial frequency patterns of linked genes may perform as barriers to the gene diffusion, and cause sharp changes of gene frequencies across the region (cf. e.g., [8]). It is suggested that barriers be a significant factor inducing the genetic diversity and specious isolation in ecological systems.

1991 Mathematics Subject Classification. 35K20, 35D05, 35B35, 35B40, 92D25.

Key words and phrases. Diffusion, Fixed Interface, Gene, Barrier, Permeability. 
In this paper, a model with two alleles $A_{1}, A_{2}$ at a specific diallelic locus is considered. Let $G \subset \mathbb{R}^{n}$ be composed of two adjacent bounded open connected sets $G_{l}, l=1,2$, separated by a surface $\Sigma=\partial G_{1} \cap \partial G_{2} \subset \mathbb{R}^{n-1}$. Here $G$ and $\Sigma$ represent respectively the isolated finite habitat and the imbedded narrow barrier (for representing a physical habitat, $n=1,2,3$. However, we do not restrict ourselves to low spatial dimensions.). Let $0 \leq u(x, t) \leq 1$ be the allele frequency of $A_{1}$ at the spatial position $x$ at time $t$. A fixed interface boundary value problem for general diffusion-reaction differential equations governing changes in $u$ is formulated as

$$
\begin{array}{rll}
u_{t}-L u=g(x, u) & \text { in } & G \times(0, T), \\
N u=0 & \text { on } & (\partial G \backslash \Sigma) \times(0, T], \\
\left.N u\right|_{\Sigma_{-}}=\lambda(x)[u]=-\left.N u\right|_{\Sigma_{+}} & \text {on } & \Sigma \times(0, T], \\
u(\cdot, 0)=u_{0} & \text { in } & G .
\end{array}
$$

In the above equation, the elliptic differential operator $L$ and the boundary operator $N$ are given by

$$
\begin{aligned}
& L u=\sum_{i, j=1}^{n} D_{i}\left(a^{i j}(x) D_{j} u\right), x \in G, \\
& N u=\sum_{i, j=1}^{n}\left(a^{i j}(x) D_{j} u\right) \nu_{i}, x \in \partial G_{l}
\end{aligned}
$$

with $\left(\nu_{1}, \ldots, \nu_{n}\right)$ the outward unit normal vector at $x \in \partial G_{l}$, and

$$
\sum_{i, j=1}^{n} a^{i j}(x) \xi_{i} \xi_{j} \geq \theta_{0}|\xi|^{2}, \forall x \in G, \xi \in \mathbb{R}^{n}
$$

for some $\theta_{0}>0$. In the interface condition $(1.1)_{3}, \lambda \geq 0$ on $\Sigma,[u]=\left.u\right|_{\Sigma_{+}}-\left.u\right|_{\Sigma_{-}}$with $\left.u\right|_{\Sigma_{ \pm}}$ the limits of $u$ at $\Sigma$ as $\Sigma$ is approached from the interior of $G_{2}, G_{1}$ respectively, and $\left.N u\right|_{\Sigma_{ \pm}}$ are the boundary derivatives of $u$ at $\Sigma$ in the outward direction of $G_{2}, G_{1}$ respectively.

Equation (1.1) is a generalization of diffusion models with interface conditions treated by Barton, Nagylaki and others (cf. e.g., $[\mathbf{2}, \mathbf{7}, \mathbf{8}]$ ) for the study of the dispersal of a gene at a diallelic locus across narrow barriers. Here by modeling the allele frequency diffusion flux with $\sum_{j=1}^{n} a^{i j}(x) D_{j} u$, equations $(1.1)_{1}-(1.1)_{2}-(1.1)_{4}$, i.e., a homogeneous Neumann boundary value problem for general diffusion-reaction equations, govern the evolution of frequency of $A_{1}$ in the population subject to natural selection away from the barrier in an isolated habitat. When across the barrier, since the sharp change of allele frequency $[u]$ is typically proportional to the allele frequency flux outside the barrier (cf. e.g., [8]), the diffusion type fixed interface condition $(1.1)_{3}$ models the transport of allele $A_{1}$ through the barrier. In this condition, $\lambda(x)$ gives the permeability of the barrier at $x \in \Sigma$. When the evolution reaches an equilibrium, $u$ satisfies the elliptic problem:

$$
\begin{array}{rll}
-L u=g(x, u) & \text { in } & G, \\
N u=0 & \text { on } & \partial G \backslash \Sigma, \\
\left.N u\right|_{\Sigma_{-}}=\lambda(x)[u]=-\left.N u\right|_{\Sigma_{+}} & \text {on } & \Sigma .
\end{array}
$$

Equation (1.1) in general models the transport of diffusing materials or heat in an isolated medium with an embedded porous thin barrier. Some results of the existence and regularity of solutions of the linear problem of (1.1) were reported in [4] (also see lemma 1 below).

The main purpose of this work is to establish physically sensible bounded solutions of (1.1) (for instance, if $u$ is the allele frequency then $0 \leq u \leq 1$ ), and criteria of stability and instability of equilibria (solutions of (1.5)) in terms of the permeability parameter $\lambda$. In section 2, we establish a comparison principle for parabolic differential equations with interface conditions. Using this new comparison principle, we prove the global existence of 
bounded solutions of (1.1) in the weak form in the framework of Hilbert space. In section 3 , we derive sufficient conditions of stability and instability of equilibria under dynamical systems generated by bounded weak solutions. Special attention is paid to the effect of $\lambda$ in the stability analysis. In section 4 , we specialize the reaction term $g$ in $(1.1)_{1}$ for discussion. Here we show that constant equilibria may change stabilities when the permeability of barrier changes through a critical value. We also present the stable nonconstant equilibria which arise as a consequence of the interaction of the selection force and the barrier. In section 5, we give an overview of this paper and remarks. Some general criteria of stability and instability of equilibria used in section 3 are given in the Appendix.

We now give notation and the weak formulation of (1.1). For $k \in \mathbb{Z}_{+}$, let $H^{k}\left(G_{1}\right) \subset$ $L^{2}\left(G_{1}\right)$ be the Sobolev space, and $C^{k}\left(\bar{G}_{1}\right)$ the space of functions with uniformly continuous partial derivatives of order $\leq k$ in $G_{1}$. Define $H^{k}=H^{k}\left(G_{1}\right) \times H^{k}\left(G_{2}\right)$, and $\|\psi\|_{H^{k}}^{2}=$ $\left\|\psi^{(1)}\right\|_{H^{k}\left(G_{1}\right)}^{2}+\left\|\psi^{(2)}\right\|_{H^{k}\left(G_{2}\right)}^{2}$ for $\psi \in H^{k}$. The space $C^{k}$ and the norm of $C^{k}$ are similarly defined. We identify a function $\psi$ in $G$ with $\left(\psi^{(1)}, \psi^{(2)}\right)$ in which $\psi^{(l)}$ is the restriction of $\psi$ to $G_{l}, l=1,2$. For $\psi_{1}, \psi_{2} \in H^{1}$, define

$$
B\left(\psi_{1}, \psi_{2}\right)=\sum_{i, j=1}^{n} \int_{G} a^{i j} D_{j} \psi_{1} D_{i} \psi_{2}+\int_{\Sigma} \lambda\left[\psi_{1}\right]\left[\psi_{2}\right],
$$

where $[\psi]=\left.\psi^{(2)}\right|_{\Sigma}-\left.\psi^{(1)}\right|_{\Sigma}$, and $\left.\psi^{(l)}\right|_{\Sigma}$ is the restriction of $\psi^{(l)}$ to $\Sigma, l=1,2$ in the sense of trace. Let $u \in L^{2}\left((0, T), H^{1}\right)$ with distributional derivative $u^{\prime} \in L^{2}\left((0, T), H^{1^{*}}\right)$, where $H^{1^{*}}$ is the dual space of $H^{1}$. We refer to $u$ as a weak solution of (1.1) if for almost all $t \in(0, T)$,

$$
\left\langle u^{\prime}(t), \psi\right\rangle+B(u(t), \psi)=(g(\cdot, u(t)), \psi)_{L^{2}}, \forall \psi \in H^{1},
$$

and $u(0)=u_{0}$ a.e. in $G$, where $\langle\cdot, \cdot\rangle$ is the action of elements in $H^{1^{*}}$ on elements in $H^{1}$, and $(\cdot, \cdot)_{L^{2}}$ is the $L^{2}$-inner product. Also, $u \in H^{1}$ is a weak equilibrium if $B(u, \psi)=(g(\cdot, u), \psi)_{L^{2}}$, $\forall \psi \in H^{1}$. We note that from the integration by parts, a weak (equilibrium) solution $u$ is a regular (equilibrium) solution of $(1.1)$ if $u \in C^{1}\left([0, T], C^{2}\right)\left(u \in C^{2}\right)$.

For convenience, we assume throughout the paper that boundaries of the spatial domain $G$ of (1.1) are smooth, $a^{i j}=a^{j i}$, and all coefficients $a^{i j}, \lambda$ are smooth in $G$ up to the boundary. The non-linearity $g(\cdot, \cdot)$ is smooth in $G \times \mathbb{R}$. Weak (equilibrium) solutions are generally referred to as (equilibrium) solutions. A result of the existence and regularity of solutions of the linear problem of (1.1) (cf. [4]) is given in the next lemma.

Lemma 1. Let $u_{0} \in L^{2}, f \in L^{2}\left((0, T), L^{2}\right)$. Then $\exists$ ! weak solution $u$ of the linear problem $u_{t}-L u=f(x, t)$ in $G \times(0, T)$ with $(1.1)_{2^{-}}(1.1)_{4}$ such that

$$
\|u\|_{L^{2}\left((0, T), H^{1}\right)}+\left\|u^{\prime}\right\|_{L^{2}\left((0, T), H^{1^{*}}\right)} \leq C\left(\left\|u_{0}\right\|_{L^{2}}+\|f\|_{L^{2}\left((0, T), L^{2}\right)}\right)
$$

for some $C>0$. If $u_{0} \in C^{\infty}, f \in C^{\infty}\left([0, T], C^{\infty}\right)$ then $u \in C^{\infty}\left([0, T], C^{\infty}\right)$.

Proof: Let $\left\{w_{k}\right\}$ be a basis for $H^{1}$ and let $c_{k} \in \mathbb{R}$ be such that $\sum_{k=1}^{m} c_{k} w_{k} \rightarrow u_{0}$ in $L^{2}$. Define $u_{m}:[0, T] \rightarrow H^{1}$ by $u_{m}(t)=\sum_{k=1}^{m} d_{k}^{m}(t) w_{k}$ such that

$$
\begin{aligned}
\left(u_{m}^{\prime}(t), w_{k}\right)_{L^{2}}+B\left(u_{m}(t), w_{k}\right) & =\left(f(t), w_{k}\right)_{L^{2}}, \\
d_{k}^{m}(0) & =c_{k}
\end{aligned}
$$

for $k=1, \ldots, m$, for $m=1,2, \ldots$ Using (1.4), by corollary 23.26 of $[\mathbf{1 1}]$, we have $u_{m} \rightarrow u$ in $L^{2}\left((0, T), H^{1}\right)$ such that $u$ is a unique weak solution of the linear problem with $(1.8)$ (see details of the proof in theorem 1 of [4]). The regularity of weak solutions results from corollary 1 of $[\mathbf{4}]$ under the condition that $L$ is symmetric.

\section{Bounded Global Solutions}

We begin with establishing a comparison principle for parabolic differential equations with interface conditions. 
Lemma 2. If $u \in C^{1}\left([0, T], C^{2}\right)$ satisfies $u_{t}-L u+d(x, t) u \leq 0$ in $G \times(0, T)$, where

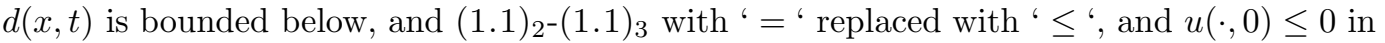
$G$ then $u \leq 0$ in $G \times[0, T]$.

Proof: Because $e^{-k t} u$ satisfies the condition satisfied by $u$ with $d(x, t)$ replaced by $k+$ $d(x, t) \geq 0$ for sufficiently large $k$, it suffices to prove the lemma with $d(x, t) \geq 0$. We let $d \geq 0$ in the following.

Assume that $u>0$ at $(x, t) \in G \times(0, T]$. It follows that

$$
u(\hat{x}, \hat{t})=\max \left\{u^{(l)}(x, t):(x, t) \in \bar{G}_{l} \times[0, T], l=1,2\right\}>0
$$

for a $(\hat{x}, \hat{t}) \in \bar{G}_{1} \times[0, T]$ or $\bar{G}_{2} \times[0, T]$. Assume $(\hat{x}, \hat{t}) \in \bar{G}_{1} \times[0, T]$. Since $d \geq 0$ and $G_{1}$ is connected with smooth boundary, from the strong maximum principle for parabolic differential operators and $u(\cdot, 0) \leq 0$ in $G,(\hat{x}, \hat{t}) \in \partial G_{1} \times(0, T]$. In view of for example, theorem 9.12 of $[\mathbf{1 0}]$, the directional derivative of $u^{(1)}$ at $(\hat{x}, \hat{t})$ in any outward direction $\nu$ of $G_{1}$ is positive, i.e., $\frac{\partial}{\partial \nu} u^{(1)}(\hat{x}, \hat{t})>0$. By $(1.4), N u^{(1)}(\hat{x}, \hat{t})>0$. From $N u \leq 0$ on $(\partial G \backslash \Sigma) \times(0, T]$ and $\left.N u\right|_{\Sigma_{-}} \leq \lambda(x)[u]$ on $\Sigma \times(0, T]$, it follows that $(\hat{x}, \hat{t}) \in \Sigma \times(0, T]$, and $0<N u(\hat{x}, \hat{t}) \leq \lambda(\hat{x})[u](\hat{x}, \hat{t})$, namely $[u](\hat{x}, \hat{t})>0$. The result contradicts against $(\hat{x}, \hat{t})$ being a maximal point of $u$ over $\bar{G}_{1} \times[0, T]$ and $\bar{G}_{2} \times[0, T]$. Therefore $(\hat{x}, \hat{t}) \notin \bar{G}_{1} \times[0, T]$. In the same manner, we show that $(\hat{x}, \hat{t}) \notin \bar{G}_{2} \times[0, T]$. Hence $u>0$ at a point $(x, t) \in G \times(0, T]$ is false. The lemma is proved.

If $\underline{u}$ (resp. $\bar{u}) \in C^{2}$ satisfies $(1.5)$ with ' $=$ ' replaced with ' $\leq$ ' (resp. ' $\geq$ ') then $\underline{u}$ (resp. $\bar{u}$ ) is a lower (resp. upper) solution for (1.5). We denote lower and upper solutions by $\underline{u}, \bar{u}$ respectively here and after in the paper.

Theorem 1. If $u_{0} \in M_{[\underline{u}, \bar{u}]}=\{\psi: \underline{u} \leq \psi \leq \bar{u}$ a.e. in $G\}$ then $\exists$ ! solution $u$ of $(1.1)$ such that $u(t) \in M_{[\underline{u}, \bar{u}]}, \forall t \in[\overline{0}, T], \forall T>0$.

\section{Proof:}

We first assume that $g_{\xi}(x, \xi)=\frac{\partial}{\partial \xi} g(x, \xi)$, where in this paper $g(x, \xi)$ is smooth respect to $x$ and $\xi$, is bounded. We show that if $u_{0} \in L^{2}$ and $\underline{u} \leq u_{0}$ a.e. in $G$ then $\exists$ ! solution $u$ of (1.1), $\forall T>0$ such that $\underline{u} \leq u(t)$ a.e. in $G, \forall t \in[0, T]$.

For $v \in X=C\left([0, T], L^{2}\right)$, define $\Psi(v)$ by a unique solution of $u_{t}-L u=g(x, v)$ in $G \times(0, T)$ with $(1.1)_{2}-(1.1)_{4}$. From (1.8) and the boundedness of $g_{\xi}, \forall T>0, \Psi: X \mapsto X$ is such that for some $C>0$,

$$
\|\Psi(u)-\Psi(v)\|_{X} \leq C T\|u-v\|_{X}, \forall u, v \in X
$$

By the contraction map principle, if $C T<1$ then $\exists$ ! $u \in X$ with $u=\Psi(u)$, namely, $u$ is a unique solution of (1.1). On the other hand, the boundedness of $g_{\xi}$ allows the interval of existence $[0, T]$ of $u$ to extend to any $T>0$. Now let $Y=\{v \in X: \underline{u} \leq v(t)$ a.e. in $G, \forall t \in$ $[0, T]\} . Y$ is a closed subset of $X$. We claim that $\Psi: Y \mapsto Y$. Therefore the unique solution $u$ lies in $Y$, i.e., $\underline{u} \leq u(t)$ a.e. in $G, \forall t \in[0, T]$, if the claim is true. We now verify the claim.

We first assume $\underline{u} \leq u_{0} \in C^{\infty}$. Let $v \in Y \cap C^{\infty}\left([0, T], C^{\infty}\right)$. By lemma 1 , we have $\underline{u}-\Psi(v) \in C^{\infty}\left([0, T], C^{2}\right)$. Since $g_{\xi}$ is bounded, $\exists \delta>0$ such that $\forall x \in G, g(x, \xi)+\delta \xi$ is an increasing function of $\xi \in \mathbb{R}$. It follows that $\underline{u}-\Psi(v)$ satisfies the condition of lemma 2 with $d=\delta$, and hence $\Psi(v) \in Y$. Namely, $\Psi: Y \bar{\bigcap}^{\infty}\left([0, T], C^{\infty}\right) \mapsto Y$. As $Y \cap C^{\infty}\left([0, T], C^{\infty}\right)$ is dense in $Y$ and $\Psi$ is continuous in $Y$, the claim is verified for this case. As $C^{\infty} \subset L^{2}$ is dense, and $u(t)$ depends on $u_{0}$ continuously in $L^{2}, \forall t \geq 0$ (we omit the proof), the result extends to $\underline{u} \leq u_{0} \in L^{2}$.

A parallel argument yields that if $g_{\xi}$ is bounded, $u_{0} \in L^{2}$, and $u_{0} \leq \bar{u}$ a.e. in $G$ then $\exists$ ! solution $u$ of (1.1) such that $u(t) \leq \bar{u}$ a.e. in $G, \forall t \in[0, T], \forall T>0$. If $g_{\xi}$ is unbounded, we replace $g$ with a smooth function $\tilde{g}$ identical to $g$ in $G \times[\min \underline{u}$, $\max \bar{u}]$, and vanishing outside a bounded open set containing $\bar{G} \times[\min \underline{u}, \max \bar{u}]$. Since $\tilde{g}_{\xi}$ is bounded, $\exists$ ! solution 
$\tilde{u}$ for this new equation with $\underline{u} \leq \tilde{u}(t) \leq \bar{u}$ a.e. in $G, \forall t \in[0, T]$. By $\tilde{g}(x, \tilde{u})=g(x, \tilde{u}), \tilde{u}$ is a unique solution of (1.1), $\forall T>0$.

Denote by $u(t ; \psi)$ the unique solution of (1.1) with $u(0)=\psi$. In view of theorem 1 , the mapping $S(t): L^{2} \mapsto L^{2}$ defined by $S(t) \psi=u(t ; \psi)$ is invariant in $M_{[\underline{u}, \bar{u}]}, \forall t \geq 0$ $\left(\{S(t): t \geq 0\}\right.$ is a dynamical system on $\left.M_{[\underline{u}, \bar{u}]}\right)$. From the remark above lemma 8 in the Appendix, $\exists$ equilibrium $\phi \in M_{[\underline{u}, \bar{u}]}$. We investigate stability and instability of $\phi$ under the established dynamical system in the following section.

\section{Stability and Instability of Equilibria}

In this section, we establish sufficient conditions of stability and instability of an equilibrium $\phi \in M_{[\underline{u}, \bar{u}]}$ on $\lambda$. Let $\mathcal{N}(r ; \phi)=\left\{\psi \in L^{2}:\|\psi-\phi\|_{L^{2}}<r\right\}$ with $r>0$. We define $\phi$ to be stable if $\forall \epsilon>0, \exists \eta>0$ such that for $\psi \in M_{[\underline{u}, \bar{u}]} \cap \mathcal{N}(\eta ; \phi), u(t ; \psi) \in \mathcal{N}(\epsilon ; \phi), \forall t \geq 0$, and unstable otherwise. Also, $\phi$ is isolated if $M_{[u, \bar{u}]} \cap \mathcal{N}(r ; \phi)$ contains no other equilibria for some $r>0$. Let $d(x)=g_{\xi}(x, \phi(x))$, and $\lambda \in \Lambda=\left\{\lambda \in C^{\infty}: \lambda^{(1)}=\lambda^{(2)} \geq 0\right.$ on $\left.\Sigma\right\}$. Incorporating the dependence of $B$ in (1.6) on $\lambda$, we define

$$
\mathcal{Q}(\psi ; \lambda)=B(\psi, \psi ; \lambda)-\int_{G} d \psi^{2} .
$$

From (1.4) and $d \in L^{\infty}$, it follows that $\mathcal{Q}(\psi ; \lambda) \geq \theta_{0}\|\psi\|_{H^{1}}^{2}-\theta_{1}\|\psi\|_{L^{2}}^{2}, \forall \psi \in H^{1}$ for some $\theta_{1}>0$, i.e., $\mathcal{Q}$ is a Gårding form in $H^{1} \subset \subset L^{2}$. Therefore $\exists$ minimizer $\varphi(\lambda) \in H^{1}$ with $\|\varphi(\lambda)\|_{L^{2}}=1$ of the minimization problem:

$$
\mu_{p}(\lambda)=\mathcal{Q}(\varphi(\lambda) ; \lambda)=\inf \left\{\mathcal{Q}(\psi ; \lambda): \psi \in H^{1},\|\psi\|_{L^{2}}=1\right\}
$$

such that $\mu_{p}(\lambda), \varphi(\lambda)$ solve the eigenvalue problem

$$
B(u, \psi)-\int_{G} d u \psi=\mu \int_{G} u \psi, \forall \psi \in H^{1}
$$

for $\mu, u$, and $\mu_{p}(\lambda)$ is the least value of $\mu$ for (3.14) to be solved by nonzero function $u$ (cf. e.g., theorem $22 . G$ of $[\mathbf{1 1}]$ ). Moreover, since $|\varphi| \in H^{1}$ and $[|\varphi(\lambda)|]^{2} \leq[\varphi(\lambda)]^{2}$ on $\Sigma$, we have that $\mathcal{Q}(\varphi(\lambda) ; \lambda)=\mathcal{Q}( \pm|\varphi(\lambda)| ; \lambda)$. Hence $\varphi(\lambda)$ can also be of one sign in $G$. We note that by a regularity argument, solutions of (3.14) are in $H^{2}$ (cf. theorem 7 of [4]). Hence $\varphi(\lambda) \in H^{2}$. We show the monotonicity and continuity of $\mu_{p}$ on $\lambda$ in the following.

Lemma 3. (i) $\mu_{p}\left(\lambda_{1}\right) \leq \mu_{p}\left(\lambda_{2}\right), \forall \lambda_{1} \leq \lambda_{2} \in \Lambda$ on $\Sigma$; (ii) $\mu_{p}$ is continuous in $\Lambda$ w.r.t. the sup-norm $\|\cdot\|_{C}$.

Proof: (i) follows from the observation that $\mathcal{Q}\left(\psi ; \lambda_{1}\right) \leq \mathcal{Q}\left(\psi ; \lambda_{2}\right), \forall \psi \in H^{1}$ if $\lambda_{1} \leq \lambda_{2}$ on $\Sigma$. For part (ii), let $\lambda_{1}, \lambda_{2} \in \Lambda$. By a simple calculation,

$$
\mathcal{Q}\left(\psi ; \lambda_{1}\right)-\mathcal{Q}\left(\psi ; \lambda_{2}\right) \leq C\left\|\lambda_{1}-\lambda_{2}\right\|_{C}\|\psi\|_{H^{1}}, \forall \psi \in H^{1}
$$

for some $C>0$. We now specialize $\psi$ to the constant function $\psi=|G|^{-1 / 2}$. For a minimizer $\varphi(\lambda)$ of $(3.13)$, by $\mathcal{Q}(\varphi(\lambda) ; \lambda) \leq \mathcal{Q}(\psi ; \lambda)=-|G|^{-1} \int_{G} d$ and the Garding form of $\mathcal{Q}$, we have $\|\varphi(\lambda)\|_{H^{1}}^{2} \leq \theta_{0}^{-1}\left(-|G|^{-1} \int_{G} d+\theta_{1}\right)$. From (3.15),

$$
\mathcal{Q}\left(\varphi\left(\lambda_{1}\right) ; \lambda_{1}\right) \leq \mathcal{Q}\left(\varphi\left(\lambda_{2}\right) ; \lambda_{1}\right) \leq C\left\|\lambda_{1}-\lambda_{2}\right\|_{C}+\mathcal{Q}\left(\varphi\left(\lambda_{2}\right) ; \lambda_{2}\right)
$$

for some $C>0$. As a result, $\left|\mu_{p}\left(\lambda_{1}\right)-\mu_{p}\left(\lambda_{2}\right)\right| \leq C\left\|\lambda_{1}-\lambda_{2}\right\|_{C}$, i.e., (ii) is proved.

Lemma 4. Assume that $\exists \lambda_{1} \leq \lambda_{2}$ such that $\mu_{p}\left(\lambda_{1}\right)<0<\mu_{p}\left(\lambda_{2}\right)$. Let $\lambda_{\alpha}=$ $(1-\alpha) \lambda_{1}+\alpha \lambda_{2}$, where $0 \leq \alpha \leq 1$. Then $\exists ! \alpha_{0} \in(0,1)$ such that $\mu_{p}\left(\lambda_{\alpha_{0}}\right)=0$ and $\mu_{p}\left(\lambda_{\alpha}\right)$ is strictly increasing at $\alpha_{0}$. 
Proof: Clearly, $\alpha \mapsto \lambda_{\alpha}$ is continuous and nondecreasing. Hence from lemma $3, \mu_{p}\left(\lambda_{\alpha}\right)$ is continuous and nondecreasing in $\alpha$. Assume that $\mu_{p}\left(\lambda_{\alpha_{1}}\right)=\mu_{p}\left(\lambda_{\alpha_{2}}\right)$ for some $0 \leq \alpha_{1}<$ $\alpha_{2} \leq 1$. By the monotonicity of $\mu_{p}\left(\lambda_{\alpha}\right)$ w.r.t. $\alpha, \mu_{p}\left(\lambda_{\alpha}\right)=\mu_{p}\left(\lambda_{\alpha_{1}}\right), \forall \alpha \in\left[\alpha_{1}, \alpha_{2}\right]$. Secondly, by $\mathcal{Q}\left(\varphi\left(\lambda_{\alpha_{2}}\right) ; \lambda_{\alpha_{2}}\right) \geq \mathcal{Q}\left(\varphi\left(\lambda_{\alpha_{2}}\right) ; \lambda_{\alpha_{1}}\right) \geq \mathcal{Q}\left(\varphi\left(\lambda_{\alpha_{1}}\right) ; \lambda_{\alpha_{1}}\right)$, it yields $\int_{\Sigma}\left(\lambda_{\alpha_{2}}-\lambda_{\alpha_{1}}\right)\left[\varphi\left(\lambda_{\alpha_{2}}\right)\right]^{2}=0$, and hence $\left[\varphi\left(\lambda_{\alpha_{2}}\right)\right]=0$ on the part of $\Sigma$ where $\lambda_{1}<\lambda_{2}$. Hence $\mu_{p}\left(\lambda_{\alpha}\right)=\mathcal{Q}\left(\varphi\left(\lambda_{\alpha_{2}}\right) ; \lambda_{\alpha}\right)$, $\forall \alpha \in\left[\alpha_{2}, 1\right]$. In summary, $\mu_{p}\left(\lambda_{\alpha}\right)=\mu_{p}\left(\lambda_{2}\right), \forall \alpha \in\left[\alpha_{1}, 1\right]$. Consequently, $\mu_{p}\left(\lambda_{\alpha}\right)$ is strictly increasing at $\alpha$ if $\mu_{p}\left(\lambda_{\alpha}\right)<\mu_{p}\left(\lambda_{2}\right)$. The lemma follows from the continuity of $\mu_{p}\left(\lambda_{\alpha}\right)$ in $\alpha$.

Lemma 5. $\mu_{p}(\lambda)>0$ in the following cases: $(i) d \leq 0, \int_{G} d<0 ;(i i) \lambda_{\alpha_{0}}<\lambda$ on $\Sigma$ with $\lambda_{\alpha_{0}}$ given in lemma 4 .

Proof: In $(i)$, if $d \leq 0$ then $\mu_{p}(\lambda)=\mathcal{Q}(\varphi(\lambda) ; \lambda) \geq B(\varphi(\lambda), \varphi(\lambda)) \geq 0$. Assume $\mu_{p}(\lambda)=0$. Then $0=B(\varphi(\lambda), \varphi(\lambda)) \geq \theta_{0}\|D \varphi(\lambda)\|_{L^{2}}^{2}$ and $\int_{G} d \varphi^{2}(\lambda)=0$. However, from the former, $\varphi(\lambda)$ is a constant, and hence it follows that $\int_{G}^{G} d \varphi^{2}(\lambda)<0$. The contradiction leads to $\mu_{p}(\lambda)>0$. In $(i i)$, because $\Sigma$ is compact and $\lambda_{\alpha_{0}}<\lambda$ are continuous on $\Sigma, \exists \alpha>\alpha_{0}$ such that $\lambda_{\alpha} \leq \lambda$ on $\Sigma$. From the strict monotonicity of $\mu_{p}\left(\lambda_{\alpha}\right)$ at $\alpha_{0}, \mu_{p}(\lambda) \geq \mu_{p}\left(\lambda_{\alpha}\right)>0$.

Lemma 6. $\mu_{p}(\lambda)<0$ in the following cases: $(i) \int_{G} d>0$, or $\int_{G} d=0, d \neq 0$ a.e. in $G ;($ ii $) \int_{G} d<0, \int_{G_{1}} d \cdot \int_{G_{2}} d<0$, and $\frac{1}{\int_{G_{1}} d}+\frac{1}{\int_{G_{2}} d}<\frac{1}{\int_{\Sigma} \lambda} ;$ (iii) $\lambda<\lambda_{\alpha_{0}}$ on $\Sigma$ with $\lambda_{\alpha_{0}}$ given in lemma 4 .

Proof: In $(i)$, if $\int_{G} d>0$ then $\mu_{p}(\lambda) \leq \mathcal{Q}(C ; \lambda)=-C^{2} \int_{G} d<0$ for $C \in \mathbb{R}$. Similarly, if $\int_{G} d=0$ then $\mu_{p}(\lambda) \leq 0$. However, if $\mu_{p}(\lambda)=0$, we have $\varphi(\lambda)=|G|^{-\frac{1}{2}}$. From the consequence below (3.13), $\int_{G} d \psi=0, \forall \psi \in H^{1}$, namely, $d=0$, a.e. in $G$. Hence $\mu_{p}(\lambda)<0$. In $(i i)$, we simplify the notation by letting $D_{1}=\int_{G_{1}} d, D_{2}=\int_{G_{2}} d$ and $W=\int_{\Sigma} \lambda$. Let $\psi=\left(\psi^{(1)}, \psi^{(2)}\right)=\left(C_{1}, C_{2}\right)$, where $C_{1} \neq C_{2} \in \mathbb{R}$. By a simple calculation, $\mathcal{Q}(\psi ; \lambda)=$ $\left(-D_{1}-D_{2}\right) C_{1}^{2} \mp 2\left|C_{2}-C_{1}\right| D_{2} C_{1}+\left(C_{2}-C_{1}\right)^{2}\left(W-D_{2}\right)$. Under the condition that $\int_{G} d<0$, we have $\mathcal{Q}(\psi ; \lambda)<0$, provided $D_{1} D_{2}<W\left(D_{1}+D_{2}\right)$. The last condition leads to the result of $($ ii $)$. (iii) follows from a similar argument to $($ ii) of lemma 5.

Theorem 2. In each of the cases of lemma $5, \phi$ is stable. In each of the cases of lemma $6, \exists \psi \in H^{2}$ such that $\mathcal{Q}(\psi ; \lambda)<0$. For the latter, $\phi$ is unstable if $\phi$ is isolated and $\phi+\epsilon \psi \in M_{[\underline{u}, \bar{u}]}$ for some $\epsilon>0$.

Proof: The first part of the theorem follows from lemma 7 and the result that the exponential stability implies the stability of an equilibrium. The second part of the theorem is from lemma 8 in the Appendix.

\section{Application}

In modeling the gene dispersal in a population, the effect of natural selection $g(x, u)=$ $s(x) h(u)$ is formulated. In this formulation, $s(x)$ represents the selective advantage $(s(x)>$ $0)$ or disadvantage $(s(x)<0)$ of $A_{1}$ over $A_{2}$ at the spatial location $x$, and the property of $h$ depends on the fitness of genotypes. When the fitness of the heterozygote $A_{1} A_{2}$ is intermediate to the homozygote $A_{1} A_{1}$ and $A_{2} A_{2}$ (e.g., the ratio of fitness of genotypes $A_{1} A_{1}, A_{1} A_{2}$ and $A_{2} A_{2}$ is $\left.1: 1-\frac{1}{2} s(x): 1-s(x)\right), h$ resembles qualitatively the logistic function $u(1-u)$ (cf. e.g., [5]). Accordingly, we in the following assume $h(0)=h(1)=0$, $h^{\prime}(0)>0, h^{\prime}(1)<0$. Thus $\phi=0,1$ are constant equilibria, and $S(\cdot)$ is a dynamical system in $M_{[0,1]}$.

Theorem 3. Assume the preceding conditions on $g$. 
(1) If either $(i) \int_{G} s \geq 0$, or (ii) $\int_{G} s<0, \int_{G_{1}} s \cdot \int_{G_{2}} s<0$, and $\frac{1}{\int_{G_{1}} s}+\frac{1}{\int_{G_{2}} s}<\frac{h^{\prime}(0)}{\int_{\Sigma} \lambda}$, then 0 is unstable, provided that 0 is isolated.

(2) If $\int_{G} s<0, \int_{G_{1}} s \cdot \int_{G_{2}} s<0$, and $h^{\prime}(0)$ is sufficiently small then $\exists$ critical value $\lambda_{0} \in \mathbb{R}_{+}$such that 0 is unstable if $\lambda<\lambda_{0}$ on $\Sigma$, provided that 0 is isolated, and exponentially stable if $\lambda_{0}<\lambda$ on $\Sigma$.

Proof: Let $\varphi(\lambda) \geq 0$ be the minimizer of (3.13) with $d(x)=h^{\prime}(0) s(x)$. Thus $\epsilon \varphi \in M_{[0,1]}$, for $\epsilon>0$ sufficiently small. By $h^{\prime}(0)>0, \int_{G_{l}} d=h^{\prime}(0) \int_{G_{l}} s$, where $l=1,2$, part 1 . of the theorem follows from theorem 2 .

To prove part 2. of the theorem, we show that $\exists 0<\lambda_{1}<\lambda_{2}$ such that $\mu_{p}\left(\lambda_{1}\right)<0<$ $\mu_{p}\left(\lambda_{2}\right)$. If what we are to show is true, the existence of the critical value $\lambda_{0}$ is then assured by lemma 4 , and part 2 of the theorem follows from theorem 2 . To begin with, we define for $\lambda \in(0, \infty)$,

$$
m(\lambda)=\inf \left\{\frac{B(\psi, \psi ; \lambda)}{\int_{G} s \psi^{2}}: \int_{G} s \psi^{2}>0, \psi \in H^{1}\right\} .
$$

It is observed that $0 \leq m\left(\lambda_{1}\right) \leq m\left(\lambda_{2}\right)$ for $\lambda_{1} \leq \lambda_{2}$, and $m$ is bounded above (we see the boundedness of $m$ by substituting a function $\psi \in H^{1}$ with $\psi \neq 0$ in $G_{1}, \psi=0$ in $G_{2}$ and $[\psi]=0$ on $\Sigma$ to the right hand side of (4.17)). We claim that $0<m(\lambda), \forall \lambda \in(0, \infty)$ if $\int_{G} s<0$.

Assume $m(\lambda)=0$ for some $\lambda \in(0, \infty)$. It follows that $\exists \psi_{k} \in H^{1}$ with $\int_{G} s \psi_{k}^{2}>0$, $\left\|\psi_{k}\right\|_{L^{2}}=1, k=1,2,3, \ldots$, such that $B\left(\psi_{k}, \psi_{k} ; \lambda\right) \rightarrow 0$ as $k \rightarrow \infty$. Passing to a subsequence if necessary, it follows that $\psi_{k} \rightarrow \hat{\psi}$ in $H^{1} \subset \subset L^{2}$, where $\|\hat{\psi}\|_{L^{2}}=1,\|D \hat{\psi}\|_{L^{2}}=0$ and $\int_{\Sigma}[\hat{\psi}]^{2}=0$, namely, $\psi=|G|^{-\frac{1}{2}}$. Therefore

$$
0<\int_{G} s \psi_{k}^{2} \rightarrow \int_{G} s \hat{\psi}^{2}=\frac{1}{|G|} \int_{G} s<0, \text { as } k \rightarrow \infty .
$$

The claim is verified by the contradiction in (4.18).

In summary, under the circumstances, $m(\cdot)>0$ is a bounded nondecreasing function in $(0, \infty)$. Thus $h^{\prime}(0)<m\left(\lambda_{2}\right)$ for some $\lambda_{2} \in(0, \infty)$ if $h^{\prime}(0)<\sup _{[0, \infty)} m$. It follows that

$$
\mathcal{Q}\left(\psi ; \lambda_{2}\right)=B\left(\psi, \psi ; \lambda_{2}\right)-h^{\prime}(0) \int_{G} s \psi^{2}>0, \forall \psi \in H^{1},
$$

and hence $\mu_{p}\left(\lambda_{2}\right)>0$. On the other hand, because $\int_{G_{1}} s \cdot \int_{G_{2}} s<0$, we may assume $\int_{G_{1}} s>0$ and $\int_{G_{2}} s<0$. Since $h^{\prime}(0)>0$, by letting $\psi=\left(\left|G_{1}\right|^{-\frac{1}{2}}, 0\right)$, we have

$$
\mathcal{Q}\left(\psi ; \lambda_{1}\right)=\left|G_{1}\right|^{-1}\left(\lambda_{1}|\Sigma|-h^{\prime}(0) \int_{G_{1}} s\right)<0
$$

for $\lambda_{1}>0$ sufficiently small, and hence $\mu_{p}\left(\lambda_{1}\right)<0$.

The stability and instability of $\phi=1$ can be obtained by a parallel argument.

Secondly, we consider a different situation that the heterozygote $A_{1} A_{2}$ is less fitted than the homozygote $A_{1} A_{1}, A_{2} A_{2}$ (e.g., $s(x)>0$, but there is 'underdominance' with the fitnesses, and the ratio of fitness of genotypes $A_{1} A_{1}, A_{1} A_{2}$ and $A_{2} A_{2}$ is given by 1 : $1-\frac{1}{2}(s(x)+\mathcal{S}(x)): 1-s(x)$ with $\left.\mathcal{S}(x)>s(x)\right)$. In this case, $g(x, u)=\mathcal{S}(x) h(u)$, and $h$ typically resembles a cubic polynomial such as $u(u-\beta)(1-u)$ with $0<\beta<1$ depending on $s, \mathcal{S}$ (cf. e.g., [8]). We hence in the following assume $g(x, 0)=g(x, 1)=0$, and $g_{\xi}(x, 0)<0$, $g_{\xi}(x, 1)<0, \forall x \in \bar{G}_{l}, l=1,2$. As in the previous case, $\phi=0,1$ are constant equilibria, and $S(\cdot)$ is a dynamical system in $M_{[0,1]}$.

Theorem 4. Assume the preceding conditions on $g$. Then $\exists$ an exponentially stable nonconstant equilibrium $\phi$ of (1.1) if $\lambda$ is sufficiently small on $\Sigma$. 
Proof: We construct nontrivial lower and upper solutions of (1.5) as follows. By the hypothesis on $g, \exists 0<\beta_{1}<\beta_{2}<1$ such that $g(x, \cdot)<0, g(x, \cdot)>0$ in intervals $\left(0, \beta_{1}\right)$ and $\left(\beta_{2}, 1\right)$, respectively, $\forall x \in G$. Let $\rho_{1} \in C^{1}\left(\bar{G}_{1}\right), \rho_{1}=0$ on $\partial G_{1} \backslash \Sigma$, and $\rho_{1} \geq 0$ but $\not \equiv 0$ on $\Sigma$. We consider a Robin type boundary value problem:

$$
\begin{array}{r}
-L u=g(x, u) \quad \text { in } \quad G_{1}, \\
N u+\frac{1}{\beta_{1}} \rho_{1}(x) u=\rho_{1}(x) \quad \text { on } \quad \partial G_{1} .
\end{array}
$$

We define for a function $v \in C^{2}\left(\bar{G}_{1}\right)$ to be a lower (resp. upper) solution of (4.21) if $v$ satisfies (4.21) with ' $=$ ' replaced by ' $\leq$ ' (resp. ' $\geq$ '). Therefore 0 and $\beta_{1}$ are lower and upper solutions of (4.21) respectively. By theorem 2.1 of [9], it follows that $\exists$ a solution $\phi_{1} \in C^{2}\left(\bar{G}_{1}\right)$ of $(4.21)$, with $0 \leq \phi_{1} \leq \beta_{1}$. We observe that $\phi_{1}$ is not constant because $\rho_{1}$ is not constant on $\partial G_{1}$. Secondly, in a parallel manner to the previous argument, we let $\rho_{2} \in C^{1}\left(\bar{G}_{2}\right), \rho_{2}=0$ on $\partial G_{2} \backslash \Sigma, \rho_{2} \geq 0$ but $\not \equiv 0$ on $\Sigma$. Replacing $\rho_{1}, G_{1}, \beta_{1}$ in (4.21) with $\rho_{2}, G_{2}, \beta_{2}$, we have that $\exists$ a nonconstant solution $\beta_{2} \leq \phi_{2} \leq 1$ for the new problem. With $\phi_{1}, \phi_{2}$, we set

$$
\underline{u}=\left\{\begin{array}{lll}
0 & \text { in } & G_{1} \\
\phi_{2} & \text { in } & G_{2}
\end{array}, \bar{u}=\left\{\begin{array}{lll}
\phi_{1} & \text { in } & G_{1} \\
1 & \text { in } & G_{2}
\end{array} .\right.\right.
$$

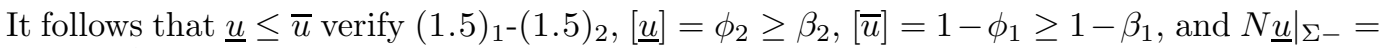
$0 \leq \rho_{2}\left(\frac{1}{\beta_{2}} \phi_{2}-1\right)=-\left.N \underline{u}\right|_{\Sigma+},\left.N \bar{u}\right|_{\Sigma-}=\rho_{1}\left(1-\frac{1}{\beta_{1}} \phi_{1}\right) \geq 0=-\left.N \bar{u}\right|_{\Sigma+}$. Consequently, $\underline{u} \leq \bar{u}$ are lower and upper solutions of (1.5) if

$$
0 \leq \lambda \leq \min \left(\frac{\rho_{1}\left(1-\frac{1}{\beta_{1}} \phi_{1}\right)}{1-\phi_{1}}, \frac{\rho_{2}\left(\frac{1}{\beta_{2}} \phi_{2}-1\right)}{\phi_{2}}\right) \text { on } \Sigma .
$$

For $\lambda$ satisfying (4.23), $\exists$ a equilibrium $\phi$ with $\underline{u} \leq \phi \leq \bar{u}$ and $[\phi] \geq \beta_{2}-\beta_{1}>0$ on $\Sigma$. Thus $\phi$ is not a constant. Since it also follows that $g_{\xi}(\cdot, \phi)<0$ in $G$, we have $\int_{G} g_{\xi}(\cdot, \phi)<0$. By lemma 5 , and lemma 7 in the Appendix, $\phi$ is exponentially stable in $M_{[0,1]}$.

\section{Discussion}

A fixed interface boundary value problem for differential equations is formulated to model the dispersal of genes across narrow barriers under natural selection. In this work, we establish for the problem the global existence of solutions, and criteria of stability and instability of equilibrium solutions. Global solutions bounded between lower and upper solutions of (1.5) are established by using a fixed point argument together with a new comparison principle for parabolic differential operator with interface condition. We derive stability and instability of equilibria as consequences of barrier permeability and selection force through spectra analysis on the linearized equation in section 3. As a result of our stability analysis, when the fitness of the heterozygote is intermediate to the homozygote, the instability of $\phi=0$ (theorem 3) indicates the allele $A_{1}$ can increase from an indefinitely low frequency if either $A_{1}$ is advantageous over $A_{2}$ in totality $\left(\int_{G} s>0\right)$, or $A_{1}$ has a local advantage over $A_{2}$ in one side of the barrier $\left(\int_{G} s<0, \int_{G_{1}} s \cdot \int_{G_{2}} s<0\right)$ with low barrier permeability $\left(\frac{1}{\int_{G_{1}} s}+\frac{1}{\int_{G_{2}} s}<\frac{h^{\prime}(0)}{\int_{\Sigma} \lambda}\right.$, or $\lambda<\lambda_{0}$ on $\left.\Sigma\right)$. Conversely, by the stability of $\phi=0$ in part 2. of theorem 3 , one sees that $A_{1}$ may not be able to increase from an indefinitely low frequency at high barrier permeability even $A_{1}$ does own a local advantage in one of the two regions separated by the barrier. When the selection is against the heterozygote (theorem 4), stable nonconstant equilibria can be reached under low barrier permeability. Because the result of theorem 4 is true for $g$ independent of $x$, it contrasts the instability of nonconstant equilibria of homogeneous Neumann boundary value problems for diffusionreaction equations (cf. $[\mathbf{3}]$ ). The inconsistence provides an evidence that the spatial patterns of gene frequency may form as a result of the joint effect of selection force and barrier. Finally, to keep the discussion simple, we have assumed that all parameters and boundaries 
of spatial domains are smooth and the differential operator $L$ is symmetric without the convection ( $1^{\text {st }}$-order derivative) terms, although these conditions can be possibly weakened. The regularity of global (weak) solutions, and bifurcation and spatial pattern formation problems relating to the barrier permeability are issues needed to be investigated in the future.

\section{Appendix}

In this section, we derive general criteria of stability and instability of equilibria.

Lemma 7. If $\mu_{p}(\lambda)>0$ then $\exists \beta, C>0$ and $\mathcal{N}(\phi ; r)$ with $r>0$ such that $\forall \psi \in$ $M_{[\underline{u}, \bar{u}]} \cap \mathcal{N}(\phi, r),\|u(t ; \psi)-\phi\|_{L^{2}} \leq C e^{-\beta t}\|\psi-\phi\|_{L^{2}}, \forall t \geq 0$, i.e., $\phi$ is exponentially stable in $M_{[\underline{u}, \bar{u}]}$.

Proof: We first mention that by a straightforward calculation, it can be verified that $S(t): L^{2} \mapsto L^{2}$ is continuously Fréchet differentiable at $\phi$ in the space of $L^{2}$, where the Fréchet derivative of $S(t)$ at $\phi$ is given by $S_{\phi}(t) u_{0}=u_{\phi}\left(t ; u_{0}\right), \forall u_{0} \in L^{2}$ with $u_{\phi}\left(\cdot ; u_{0}\right)$ a unique solution of $u_{t}-L u=g_{\xi}(x, \phi)$ in $G \times(0, T)$ with $(1.1)_{2}-(1.1)_{4}$. Since nonzero solutions $w$ of (3.14) supply an orthonormal basis for $L^{2}$ (cf. e.g., theorem 1 of [4]) and $S_{\phi}(t) w=e^{-\mu t} w$, it follows that $\left\|S_{\phi}(t)\right\|=\sup \left\{\left\|S_{\phi}(t) \psi\right\|_{L^{2}}:\|\psi\|_{L^{2}}=1\right\} \leq e^{-\mu_{p}(\lambda) t}$. The lemma is therefore true from the principle of linearized stability of equilibria with $0<\beta<\mu_{p}$ (cf. e.g., theorem 11.22 of [10]).

We present a simple argument that yields the existence of equilibria in $M_{[\underline{u}, \bar{u}]}$ in the following. Let $u$ be a solution of (1.1) with $u_{0} \in H^{2} \cap M_{[\underline{u}, \bar{u}]}$. It can be verified that $u \in L^{2}\left((0, T), H^{2}\right), u^{\prime} \in L^{2}\left((0, T), H^{1}\right)$ by using a regularity result of solutions for linear problems of (1.1) in theorem 2 of [4]. Let $J(t)=B(u(t), u(t))$. Using the integration by parts, $J^{\prime}(t)=2\left(u^{\prime}(t),-L u(t)\right) \leq-\|L u(t)\|_{L^{2}}^{2}+\|g(\cdot, u(t))\|_{L^{2}}^{2}$. Thus $J^{\prime}(t)+J(t) \leq C$ for almost all $t \in[0, T]$ for some $C>0$ depending on $\underline{u}, \bar{u}$. By integrating, we have $\theta_{0}\|D u(t)\|_{L^{2}}^{2} \leq J(t) \leq J(0)+C\left(1-e^{-t}\right), \forall t \in[0, T], \forall T>0$, and hence $\{u(t): t \geq 0\}$ is bounded in $H^{1} \subset \subset L^{2}$. As a result, by e.g., lemma 4 of $[\mathbf{6}]$, the $\omega$-limit set $\omega\left(u_{0}\right)=$ $\bigcap_{r \geq 0} \overline{\left\{S(t) u_{0}: t \geq r\right\}}$ is nonempty, compact, and connected in $L^{2}$. Moreover, $\omega\left(u_{0}\right)$ is invariant under $\{S(t), t \geq 0\}$ and $\operatorname{dist}_{L^{2}}\left(S(t) u_{0}, \omega\left(u_{0}\right)\right) \rightarrow 0$ as $t \rightarrow \infty$. Let $\mathcal{G}(x, \xi)=\int_{0}^{\xi} g(x, s) d s$. Define

$$
\mathcal{E}(\psi)=\frac{1}{2} B(\psi, \psi)-\int_{G} \mathcal{G}(\cdot, \psi),
$$

and $e(t)=\mathcal{E}(u(t))$. Thus $e^{\prime}(t)=-\left\|u^{\prime}(t)\right\|_{L^{2}}^{2} \leq 0$ for almost all $t>0$, and hence $e$ is nonincreasing in $\mathbb{R}_{+}$. Namely, $\mathcal{E}$ is a Liapunov function for $S(\cdot)$ on $M_{[u, \bar{u}]}$, and hence $\mathcal{E}(\psi)=\alpha \in \mathbb{R}, \forall \psi \in \omega\left(u_{0}\right)$ by the LaSalle invariance principle (cf. p. 234 of [1], [6]). It follows that $e^{\prime}(t)=0, \forall t>0, \forall \psi \in \omega\left(u_{0}\right)$, i.e., $\omega\left(u_{0}\right)$ consists of equilibria.

Lemma 8. Let $\phi \in M_{[\underline{u}, \bar{u}]}$ be an isolated equilibrium. If $\exists \psi \in H^{2}$ such that $\phi+\psi \in$ $M_{[\underline{u}, \bar{u}]}$ and $\mathcal{Q}(\psi)<0$ then $\exists \underline{\mathcal{N}}(r ; \phi)$ with $r>0$ such that $\forall \phi_{\epsilon}=\phi+\epsilon \psi \in \mathcal{N}(r ; \phi), \exists t_{\epsilon}>0$ such that $S(t) \phi_{\epsilon} \notin \mathcal{N}(r ; \phi), \forall t>t_{\epsilon}$.

Note that because $\phi_{\epsilon} \in M_{[\underline{u}, \bar{u}]}, \forall 0 \leq \epsilon \leq 1$, the lemma implies that $\phi$ is unstable.

Proof: Let $r^{\prime}>0$ be such that $\mathcal{N}\left(r^{\prime} ; \phi\right)$ contains no other equilibria in $M_{[\underline{u}, \bar{u}]}$. From $\mathcal{E}\left(\phi_{\epsilon}\right)=\mathcal{E}(\phi)+\frac{\epsilon^{2}}{2} \mathcal{Q}(\psi)+o\left(\epsilon^{2}\|\psi\|_{H^{1}}^{2}\right)<\mathcal{E}(\phi), \forall \epsilon>0$ sufficiently small, and $\omega\left(\phi_{\epsilon}\right)$ consisting of equilibria, we have $\mathcal{N}\left(r^{\prime} ; \phi\right) \cap \omega\left(\phi_{\epsilon}\right)=\emptyset$. Let $r=r^{\prime} / 2$. The lemma follows from $\operatorname{dist}_{L^{2}}\left(S(t) \phi_{\epsilon}, \omega\left(\phi_{\epsilon}\right)\right) \rightarrow 0$ as $t \rightarrow \infty$. 
Acknowledgement The author would like to thank Professor Paul Fife at the University of Utah for many valuable communications and suggestions during the development of this work. The author would also like to thank the anonymous reviewer for the valuable comments on this paper. This work was supported by grants of National Science Council (NSC 93-2115-M-005-007), Taiwan.

\section{References}

[1] H. Amann, Ordinary Differential Equations. An Introduction to Nonlinear Analysis, Walter de Gruyter, New York, 1990

[2] N. H. Barton, Gene flow past a cline, Heredity, 43 (1979a), pp. 333-339.

[3] R. G. Casten and C. J. Holland, Instability results for reaction diffusion equations with Neumann boundary conditions, Journal of Differential Equations, 27 (1978), pp. 266-273.

[4] C-K Chen, A barrier boundary value problem for parabolic and elliptic equations, Communications in Partial Differential Equations, 26(7 \& 8) (2001), pp. 1117-1132.

[5] W. H. Fleming, A selectiong-migration model in population genetics, Journal of Mathematical Biology, 2 (1975), pp. 219-233.

[6] J. HALE, Dynamical system and stability, Journal of Mathematical Analysis and Applications, 26 (1969), pp. 39-59.

[7] T. Nagylaki, The diffusion model for migration and selection, Some Mathematical Questions in Biology: Models in Population Biology, (1989), pp. 55-75.

[8] J. Pialek and H. H. Barton, The spread of an advantageous allele across a barrier: The effects of random drift and selection against heterozygotes, Genetics, 145 (1997), pp. 493-504.

[9] D. Sattinger, Monotone methods in nonlinear elliptic and parabolic boundary value problems, Indiana University Mathematics Journal, 21, No. 11 (1972), pp. 979-1000.

[10] J. Smoller, Shock Waves and Reaction-Diffusion Equations, Springer-Verlag, New York, 1983.

[11] E. ZeidleR, Nonlinear Functional Analysis and Its Applications II/A, Springer-Verlag, New York, 1990.

Applied Mathematics Department, National Chung-Hsing University, Taiwan.

E-mail address: cchen@amath.nchu.edu.tw 